\title{
Retained Biospecimen Description
}

National Cancer Institute

\section{Source}

National Cancer Institute. Retained Biospecimen Description. NCI Thesaurus. Code

C126072.

A textual description of the specimen types, which are collected during the course of the study. 